\title{
La guerre «blanche » des Italiens durant la Grande Guerre ou les enjeux complexes d'une guerre en montagne : adaptation, exploitation, représentation
}

Hubert Heyriès

\section{OpenEdition}

\section{Journals}

Édition électronique

URL : https://journals.openedition.org/geohist/955

DOI : $10.4000 /$ geohist.955

ISSN : 2264-2617

Éditeur

Association française de la Revue de géographie historique

Référence électronique

Hubert Heyriès, «La guerre « blanche » des Italiens durant la Grande Guerre ou les enjeux complexes d'une guerre en montagne : adaptation, exploitation, représentation », Revue de géographie historique [En ligne], 10-11 | 2017, mis en ligne le 20 mai 2017, consulté le 21 septembre 2021. URL : http:// journals.openedition.org/geohist/955; DOI : https://doi.org/10.4000/geohist.955

Ce document a été généré automatiquement le 21 septembre 2021.

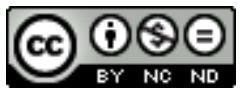

Ce(tte) œuvre est mise à disposition selon les termes de la Licence Creative Commons Attribution Pas d'Utilisation Commerciale - Pas de Modification 4.0 International. 


\title{
La guerre « blanche » des Italiens durant la Grande Guerre ou les enjeux complexes d'une guerre en montagne : adaptation, exploitation, représentation
}

\author{
Hubert Heyriès
}

1 Au cours de la Grande Guerre, sur le front italien, une guerre montagneuse se déroula entre 2000 et 3000 mètres d'altitude, rapidement baptisée "guerre blanche », car la neige était abondante. La configuration italienne du théâtre des opérations courrait en effet sur $600 \mathrm{~km}$, du Stelvio (à la frontière italo-austro-suisse) à la mer mais le relief de montagnes représentait les deux tiers de la ligne de front. Les Italiens connaissaient la guerre en montagne. Au cours des siècles, les forces du duc de Savoie puis celles du royaume de Piémont-Sardaigne s'étaient battues dans les Alpes, lors des guerres de Succession d'Espagne et d'Autriche et encore entre 1792 et 1796 contre les Français. Puis au XIXe siècle, au moment des guerres du Risorgimento, les Piémontais et les Chemises rouges garibaldiens avaient évolué en 1848 et en 1859 dans la région des lacs alpins en Lombardie. En 1866, un corps de volontaires de plus 30000 hommes commandé par Giuseppe Garibaldi avait affronté les chasseurs tyroliens autrichiens dans le Trentin. Puis les troupes de montagnes, ou Alpini, créées par le décret royal du 15 octobre 1872 avaient connu leur baptême du feu en Afrique orientale entre 1885 et 1896, en menant des opérations en altitude mais dans des conditions bien différentes de celles des Alpes, leur zone de prédilection. Pour autant, jamais les combats n'avaient connu une grande intensité, et jamais ils ne s'étaient déroulés l'hiver. Or au cours de la Grande Guerre, le commandement italien dut adapter la tactique et les modes de combat à la géographie et aux contraintes du milieu ${ }^{1}$. En fut-il capable et comment s'y prit-il ? Les diari storici, les circulaires du haut commandement italien (le Comando supremo), les souvenirs, les journaux, la littérature et le cinéma ainsi que les marques mémorielles encore visibles aujourd'hui (ossuaires et sanctuaires) apportent quelques 
éléments de réponse. Mais ces sources contribuent également à donner une représentation sacrificielle ou sacralisée du combat en montagne. Pourquoi et de quelle manière? En d'autres termes, la guerre «blanche » nécessita une adaptation au milieu qui généra une exploitation polymorphe et une représentation fantasmée de la guerre.

\section{UNE ADAPTATION AU MILIEU NECESSAIRE}

\section{Document 1 : Les régions du nord-est de l'Italie, du Trentin et de l'Istrie ${ }^{2}$}

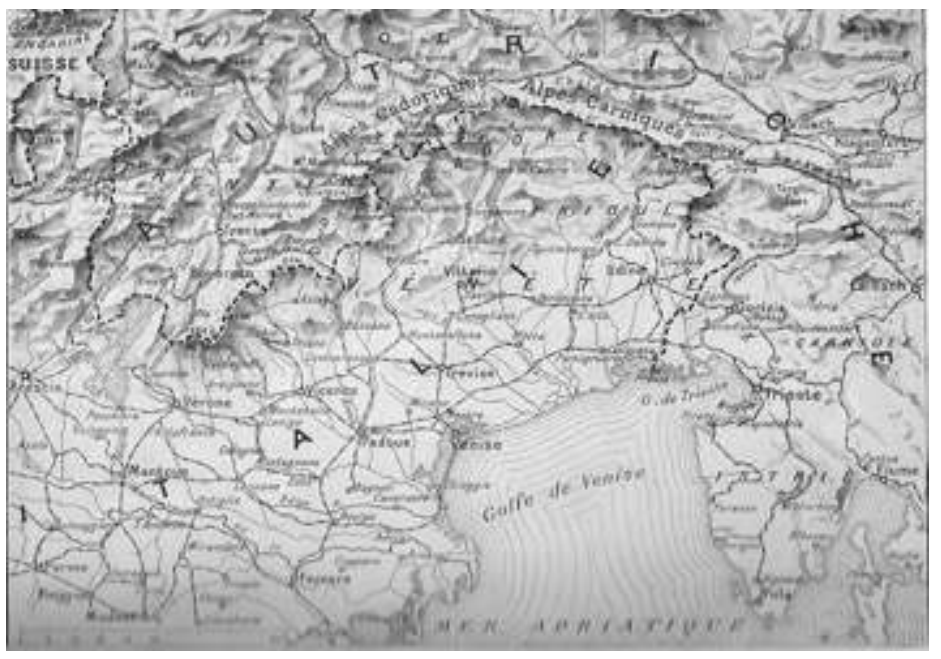

2 Le front montagnard suivait grossièrement un $\mathrm{S}$ articulé autour de deux saillants orientés en sens inverse l'un de l'autre: le Trentin et le Cadore-Frioul ${ }^{3}$. Le Trentin, dirigé vers le sud, amenait l'Autriche à quelques kilomètres de Vérone. Bloc montagneux, il s'organise de part et d'autre de la vallée de l'Adige. À l'ouest, un relief de haute montagne suit une ligne nord-sud allant du massif de l'Ortles au nord, tout ruisselant de glace et culminant à 3905 mètres, aux crêtes neigeuses de la Presanella (3 561 mètres) et au vaste plateau glacé de l'Adamello (3 554 mètres) de 3000 ha environ plus au sud. À l'est du Trentin, un autre formidable rempart est formé des monts Lessini, dominant la plaine vénitienne ne dépassant pas les 2300 mètre. Puis viennent les crêtes aériennes de la chaîne dolomitique de la Brenta (3 155 mètres) dominant du côté italien, le plateau d'Asiago et enfin les crêtes de la Marmolada culminant à 3344 mètres. Tout autour de ces puissantes saillies, disposées en groupes isolés, des cimes de 1500 à 2000 mètres s'abaissent par gradins vers la plaine du Pô avec des montagnes molles, toutes couvertes d'alpages et de forêts. Le Cadore-Frioul constitue le saillant italien. Pointant vers le nord, il étend l'Italie jusqu'à la crête des Alpes Cadorique, Carniques et Juliennes, dominant les vallées autrichiennes de la Drave et de la Save. Il s'étend à l'est des Dolomites, autour des sources de la Piave (Cadore) et du bassin du Tagliamento (Frioul). Aucune cime des Alpes Carniques et Juliennes n'arrive à 3000 mètres. Le Terglou, point culminant des Alpes Juliennes, est la dernière saillie des Alpes à l'extrême est de la zone montagneuse. Il domine la Save mais ne dépasse pas 2864 mètres. Au-delà, les formes de terrain s'émoussent débouchant sur une architecture tabulaire formée de plateaux monotones où les rivières se perdent sous terre: le fameux Karst ou Carso, à l'est de l'Isonzo, mais il s'agit d'un autre front. 
3 Ce massif montagneux n'est franchissable qu'en de rares endroits particulièrement bien fortifiés par un réseau de forts construits entre 1871 et 1914 aussi bien du côté autrichien que du côté italien ${ }^{4}$. Dans le Trentin, les routes du Stelvio (empruntant la Valteline), du Tonale et de la Giudicaria (haute vallée de l'oglio), ainsi que du val Sugana (haute vallée de la Brenta) permettent de pénétrer dans la vallée de l'Adige par l'ouest et par l'est. Dans le Cadore, une route latérale suit la haute vallée de la Piave par Auronzo. Plus à l'est, aucun col carrossable ne franchissait les Alpes Carniques. Pour autant, relief montagneux et fortifications déterminèrent la tactique italienne fondamentalement défensive jusqu'à la Grande Guerre.

4 Jusqu'en 1914 en effet, la doctrine d'emploi des forces des troupes alpines ne fut jamais très développée. Le "Règlement d'instruction et de service interne pour l'Infanterie ${ }^{5}$, datant de 1892, laissait aux Alpini toute latitude pour s'adapter aux conditions du terrain en montagne sans précision. Puis l'importance des Alpes devint telle que la doctrine d'emploi des forces de montagne fut remodelée dans les années $1900^{6}$. En 1914, les huit régiments Alpini (52 bataillons, 192 compagnies), réunis en 1910 en trois brigades, normalement armées de mitrailleuses, constituèrent les avant-gardes mobiles (avec des détachements de skieurs créés officiellement en 1902) occupant les fortifications qui verrouillaient les voies de passage. L'artillerie de montagne (trois régiments) et l'artillerie des forteresses les soutiendraient pour mener des opérations défensives ou retardataires permettant de concentrer des troupes dans la plaine padane. Par ailleurs, les Alpini avaient l'habitude de passer l'hiver dans leurs villages et les brigades n'avaient ainsi aucune expérience de la guerre en montagne l'hiver. Et l'on ne pensa à camoufler les hommes qui portaient un uniforme gris, un sac tyrolien et un chapeau mou en pain de sucre avec une plume que pendant la guerre lorsque des surtouts blancs furent distribués pour les dissimuler sur la neige.

5 À partir de 1914, les choses changèrent. Le général Luigi Cadorna, chef d'État-major général (le futur Comando supremo) adopta en effet, dans un mémoire daté du 21 août 1914, une tactique dans les Alpes, à la fois défensive et offensive ${ }^{7}$. La guerre devait désormais se faire été comme hiver et l'objectif fut de contrôler les sommets comme le précisa encore la circulaire $n^{\circ} 2324$ du Comando supremo en date du 29 avril 1917 : « L'art des fortifications se soumet au terrain, et non le terrain à l'art. [...] Pour obtenir un front le moins développé avec la plus grande économie de forces, la défense doit être portée sur les hauteurs " avec des flanquements, occupées par des mitrailleuses installés dans des cavernes creusées dans la roche et des "raccordements" (allacciamenti) ou cortine passant par les vallées, sous la forme de tranchées ${ }^{8}$. Encore fallait-il s'emparer de ces hauteurs au moyen de l'offensive frontale.

6 Cette tactique, à la fois défensive et offensive, généra une guerre polymorphe adaptée et inadaptée au milieu.

\section{UNE EXPLOITATION POLYMORPHE}

7 Les ordres d'occuper les cols et les sommets donnèrent naissance à une guerre de coups de main et d'exploits menées par de petites unités (escouade ou compagnie). Dans cette façon de faire la guerre, les alpini devinrent les troupes d'élite. Le plus célèbre des coups de main exploitant au mieux le milieu physique fut sans nul doute la prise du Monte Nero le 16 juin 1915, à 2245 mètres, dans les Alpes juliennes, objectif militaire pour s'assurer une meilleure position dans la cuvette de Caporetto et pour 
continuer l'action vers Tolmino'. Six compagnies alpini des bataillons Exilles et Susa prirent d'assaut le mont de nuit, après avoir grimpé une paroi rocheuse à pic (la face sud-est du Vrata, à 2014 mètres), pour atteindre une position d'où elles s'élancèrent à la baïonnette et à la grenade, prenant par surprise les Autrichiens. Ces épisodes restaient cependant rares, car la guerre blanche fut surtout une guerre de position ou une guerre de l'intendance et du génie plus qu'une guerre opérationnelle. Les tranchées en montagne conformément à la circulaire $\mathrm{n}^{\circ} 250$ du 10 février $1915^{10}$ devaient être soutenues par une artillerie lourde, des canons de $149 \mathrm{~mm}$ et de $210 \mathrm{~mm}$, installés de préférence sur les flancs ou au sommet des angles rentrants, en terrain dominant. Il fallait également ravitailler l'hiver les troupes restées en altitude, aussi bien en nourriture, qu'en armement, matériel et renforts. Or les accès faisaient défauts et il fallut en définitive tout créer.

8 Ce fut le tour de force du génie qui sut aménager $6500 \mathrm{~km}$ de routes (dont des routes dans les flancs des montagnes) et $700 \mathrm{~km}$ de chemin de fer type Décauville, construire des téléphériques dont celui de Enego au Monte Tomba long de $40 \mathrm{~km}$, aménager des abris creusés dans la roche et des baraques en altitude pour abriter les hommes, surtout à partir de 1916. Ce dont les troupes avaient besoin fut ainsi transporté par camions lorsque les routes le permettaient, puis à l'aide de mulets (le canon de montagne démontables en cinq parties, pouvait être porté par quatre mulets) et enfin à la force des bras, notamment pour les canons de 149 et 210 avec les munitions (une batterie de six canons italiens de $149 \mathrm{~mm}$ se trouvait ainsi au sommet du Tofana) ${ }^{11}$.

Document 2 : Une vire en haute montagne (étroite corniche sur une paroi rocheuse) ${ }^{12}$

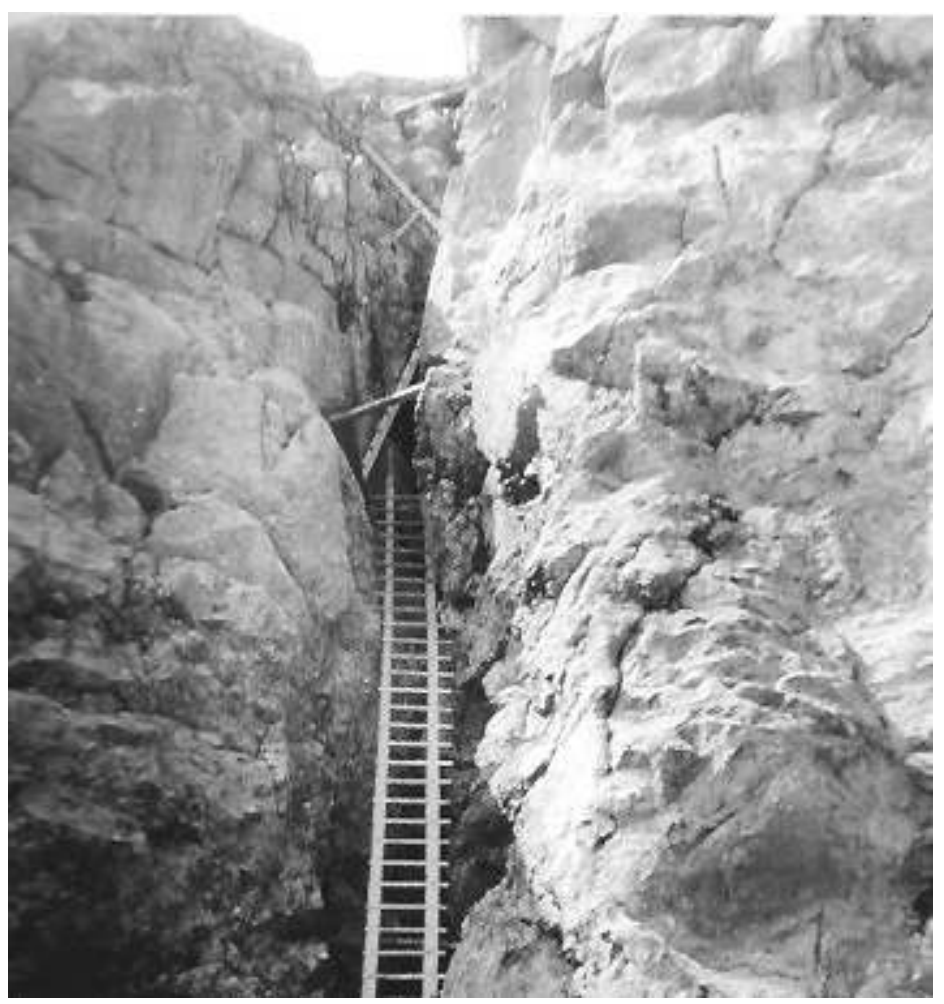

9 Au pied se trouvent les baraquements, les tranchées et les barbelés italiens ${ }^{13}$. Or cette guerre «blanche » ne fut pas menée uniquement par des alpini, mais par des centaines de milliers de fantassins qui bien souvent voyaient pour la première fois de leur vie, les 
Alpes. Mal équipés et mal entraînés, ils firent ce qu'ils purent en étant commandés par des officiers qui ignoraient la guerre en montagne et qui appliquèrent la guerre qu'ils pratiquaient sur l'Isonzo. Le témoignage inédit publié en $2008 \mathrm{du}$ fantassin volontaire Alfredo Ortali, de la $3^{\mathrm{e}}$ compagnie du $51^{\mathrm{e}}$ régiment d'infanterie de la brigade des Alpes, qui se battit dans les Dolomites entre juillet et octobre $1915^{14}$, permet de se faire une idée de la guerre type du fantassin en montagne. Alfredo Ortali alterna des périodes de repos à l'arrière-front et des périodes de huit jours de tranchées à 2000-3000 m. (dans la Marmolada et dans le secteur du col di Lana), à l'air libre ou à l'abri de médiocres baraquements où les poux et la vermine pullulaient. À l'arrière, il effectua des corvées, des tours de garde, des transports de munitions et de canons de 149 et de $210 \mathrm{~mm}$, des patrouilles en haute montagne notamment au Sasso Bianco à $2407 \mathrm{~m}$., soit plus de mille mètres de déniveler. Le tout sans aucune période d'acclimatation, sans entraînement, sans matériel adapté ni chaussure de montagne. Si l'on excepte les patrouilles en haute montagne, sa vie ressemblait fort à celle d'un camarade dans les tranchées de l'Isonzo. Le champ de bataille en montagne ressemblait en effet à celui du Carso. Au pied du col di Lana, le sous-lieutenant Gelasio Caetani décrivit ainsi le 4 novembre 1915, le champ de bataille à $2000 \mathrm{~m}$. d'altitude: "De jour, j'ai pu étudier et même dessiner ce labyrinthe de tranchées et de chemins, j'ai pu déambuler à travers ce champ de bataille tout bouleversé par les bombardements, parsemé de cadavres et couvert de fusils, de sacs à dos, de capotes, et de choses en tout genre. [...] Nous passons au milieu de barbelés et de chevaux de frise, rompus et ravagés par les tirs d'artillerie $»^{15}$.

Pour autant, la "guerre blanche" avait aussi ses propres caractères et la "mort blanche » inquiétait en permanence. La neige tombait en effet en abondance, et pouvait atteindre des épaisseurs de 4 à $5 \mathrm{~m}$ en altitude en étant permanente jusqu'à 200 jours par an. Les avalanches constituaient alors une sorte d'épée de Damoclès. Elles étaient destructrices. Celle du 13 décembre 1916, sur les pentes du Tofana, dans les Dolomites, déplaça quatre millions de mètres cubes de neige et bloqua la route d'accès à la chaîne de montagne au point qu'il fallut faire une tranchée de dix-huit mètres de haut pour rétablir la circulation. Une centaine d'artilleurs périrent dans l'avalanche, mais leur corps ne fut retrouvé qu'en mai-juin 1917, à la fonte des neiges ${ }^{16}$. Sur le glacier de l'Adamello, où les combats firent rage, un chasseur alpin du bataillon Edolo se souvint qu'à cause des chutes de neige, il était resté bloqué avec « quelques réserves de vivres, mais peu de bois ». Aussi avait-il dû se contenter de mâchonner un morceau de pain à demi geler, avec une boîte de viande que l'on devait mettre dans sa poche pour la faire dégeler et de boire du vin "distribué en morceau » avant d'être écrasé puis mis dans une bouteille, « la chaleur animale le dégelant la nuit »" Le froid fut une préoccupation permanente. Les températures en haute montagne, en moyenne entre $-5^{\circ}$ et $-20^{\circ}$ pouvaient descendre à $-35^{\circ},-40^{\circ}$. Les gelures aux mains et aux pieds furent donc fréquentes, et les hommes ne disposaient pratiquement pas de remèdes sauf du gras et des pommades que l'on passait sur les mains et les pieds. Mais il était difficile de se réchauffer, y compris dans les baraquements, car le bois manquait toujours pour entretenir un bon feu. Or les chaussures s'usèrent rapidement. Après quelques semaines de courses en montagne et d'escalade, elles étaient bonnes à être jetées : les clous s'en allaient, l'empeigne (la partie supérieure de la chaussure) laissait passer l'eau, les semelles se détachaient au moindre effort ${ }^{18}$. Aussi les gelures aux pieds furent fréquentes.

11 En outre, la tactique de l'assaut frontal, totalement inadaptée au terrain provoqua des hécatombes. D’une manière générale, les Autrichiens contrôlaient les hauteurs, et les 
Italiens les fonds de vallée. Il fallait donc partir à l'assaut des sommets en gravissant les pentes abruptes des versants et cela bien souvent à découvert. Ainsi les attaques de la brigade des Alpes, à l'automne 1915, pour prendre le col di Lana bien fortifié par les Autrichiens furent si sanglants que les hommes finirent par surnommer le sommet, « le col di sangue ». Les états-majors lancèrent en effet des offensives massives sans tenir compte des contraintes du milieu montagneux. Lors de la Strafexpedition (l'expédition punitive) en mai-juin 1916, les Austro-hongrois attaquèrent avec quatorze divisions dans le Trentin contre dix divisions italiennes ${ }^{19}$. L'année suivante, l'offensive italienne sur le massif de l'Ortigara du 10 au 29 juin 1917 employa douze divisions d'infanterie et vingt-quatre bataillons alpini, soit plus de 300000 hommes, 500 canons moyens, 450 légers et 550 bombardes pour un front de $14 \mathrm{~km}$ à peine, à une altitude oscillant entre 1000 et 2000 mètres $^{20}$. Les pertes furent considérables. La Strafexpedition coûta ainsi 147600 Italiens et 82900 Austro-hongrois ${ }^{21}$. L'Ortigara coûta aux Italiens entre 25000 et 28000 hommes et la moitié des alpini ${ }^{22}$. L'artillerie lourde et moyenne n'avait pu suivre la progression des troupes. L'artillerie de montagne s'était révélée incapable de briser les lignes de défense. Un bombardement en altitude devait tenir compte de la plus faible densité de l'air que dans la plaine, ce qui avait une incidence sur la balistique et la précision des tirs. Or, il fallait toucher des nids de canons et de mitrailleuses masqués dans la roche, des cavernes, des galeries, des postes d'observation. Un tir mal ajusté ne servait à rien ce qui était souvent le cas. Les positions défensives montagneuses se révélèrent en définitive imprenables, fussent-elles autrichiennes ou italiennes.

13 Dès lors, les positions apparurent si fortes et si imprenables que la guerre dans les Alpes devint aussi une guerre souterraine de mines, faite de sapes et de contre-sapes, surtout à partir de 1916. Le col di Lana fut pris par l'infanterie et non par les alpini dans la nuit du 17 au 18 avril 1916 grâce à l'explosion d'une mine de cinq tonnes d'explosif placés au bout d'une galerie de 130 mètres $^{23}$. Dans le Tofana, à partir de février 1916, les Italiens creusèrent en plein milieu de la paroi glacée de la montagne une galerie de 500 mètres à l'aide de deux perceuses pneumatiques hissées à la force des bras et à l'aide de câbles. Le 11 juillet suivant, l'explosion de trente-cinq tonnes d'explosifs provoqua un entonnoir de 80 mètres de diamètre et détruisit les défenses et les abris autrichiens ${ }^{24}$. Ailleurs, dans le massif du Pasubio, le massif des deux Dents présenta la particularité d'avoir, face à face, une Dent italienne et une Dent autrichienne séparé par un col. Les hommes finirent par s'enterrer dans des galeries et des abris spacieux capables de résister à des tirs d'artillerie comme dans la colline de Vauquois en France. Une guerre de mine et de contre-mine commença à partir d'août 1917 mais n'eut aucun résultat ${ }^{25}$. Parfois aussi, des galeries furent creusées à l'intérieur des glaciers comme ce fut le cas dans le massif de la Marmolada où une véritable « ville de glace " naquit ${ }^{26}$.

14 Cette guerre polymorphe déterminée en grande partie par la nature du relief et par les contraintes du milieu, fût-elle adaptée ou inadaptée, finit toutefois par nourrir une représentation fantasmée.

\section{UNE REPRESENTATION FANTASMEE}

15 La propagande s'empara en effet de cette guerre "blanche " pour idéaliser la guerre évitant ainsi d'évoquer les boucheries du Carso et de la Bainsizza. Le service 
cinématographique et photographique aux armées joua un rôle déterminant ${ }^{27}$. Le Comando supremo poussa en ce sens. L'instruction « Normes techniques et d'emploi du service photographique terrestre et aérien" rédigée en mai 1918 indiquait en effet: «Les appareils photographiques trouvent leur meilleur emploi dans les régions de haute montagne " car « l'air doit être très transparent, frais avec peu de vapeur d'eau en suspension et avec une température uniforme $»^{28}$. De fait les unités de montagne furent toujours en nombre plus importantes que les unités de campagne (4 sur 7 en 1915, puis 5 sur 8 en 1917). Les opérateurs surent traduire dans leurs images le courage, le sacrifice, la dangerosité du théâtre des opérations, mais aussi la vie quotidienne, les privations, les conditions de vie extrême en altitude. Ils n'hésitaient pas à monter au sommet des crêtes et des cols avec leur matériel pour prendre les photos en pleine montagne!

16 Ainsi le paysage alpin, spectaculaire, fut l'un des sujets préférés de la photographie de guerre italienne. Le journal italien L'Illustrazione italiana en fit souvent sa une de manière à transformer la guerre en un conflit idéalisé nourrissant les rêves de gloire dans des paysages somptueux. Le public pouvait aussi, à l'occasion d'exposition officielle comme à Rome en 1917 et à Londres en 1918, communier avec les soldats sur tous les fronts, et en particulier dans les Alpes, notamment avec les alpini. Ces derniers devinrent naturellement les héros de cette guerre de montagne fantasmée. Leur nombre ne cessa de croître passant de 26 bataillons en 1915 à 85 bataillons en 1917 avant de redescendre à 58 bataillons après Caporetto ${ }^{29}$. Leur esprit de corps était réputé en raison d'un recrutement territorial dans les vallées alpines (alors que le recrutement était national pour toutes les autres armes), et du fait d'un meilleur encadrement d'officiers de réserve issus de la bourgeoisie urbaine qui pratiquait l'alpinisme. Or les alpini furent transfigurés en héros et nourrirent un mythe ${ }^{30}$. Le grand irrédent Cesare Battisti, qui déserta l'armée autrichienne pour s'engager dans les alpini devint en effet une figure majeure de la martyrologie alpine. Lors de l'attaque du monte Corno, dans le massif du Pasubio, il fut capturé le 10 juillet 1916, puis pendu deux jours plus tard dans les fosses du château de Trente. L'indignation de l'opinion publique italienne fut générale. Son sacrifice patriotique ne faisait que confirmer la dimension surhumaine de l'alpino. Dans la presse populaire, le dessinateur Gennaro d'Amato dans l'Illustrazione italiana représenta un chasseur alpin arrachant le poteau frontière du Trentin, le transfigurant en soldat conquérant, résolu dans l'action. 
Document 3 : « Les « alpini » renversent le poteau frontière, sur une toute du Trentin. Dessin de G. d'Amato. Premières visions de guerre par les artistes italiens.

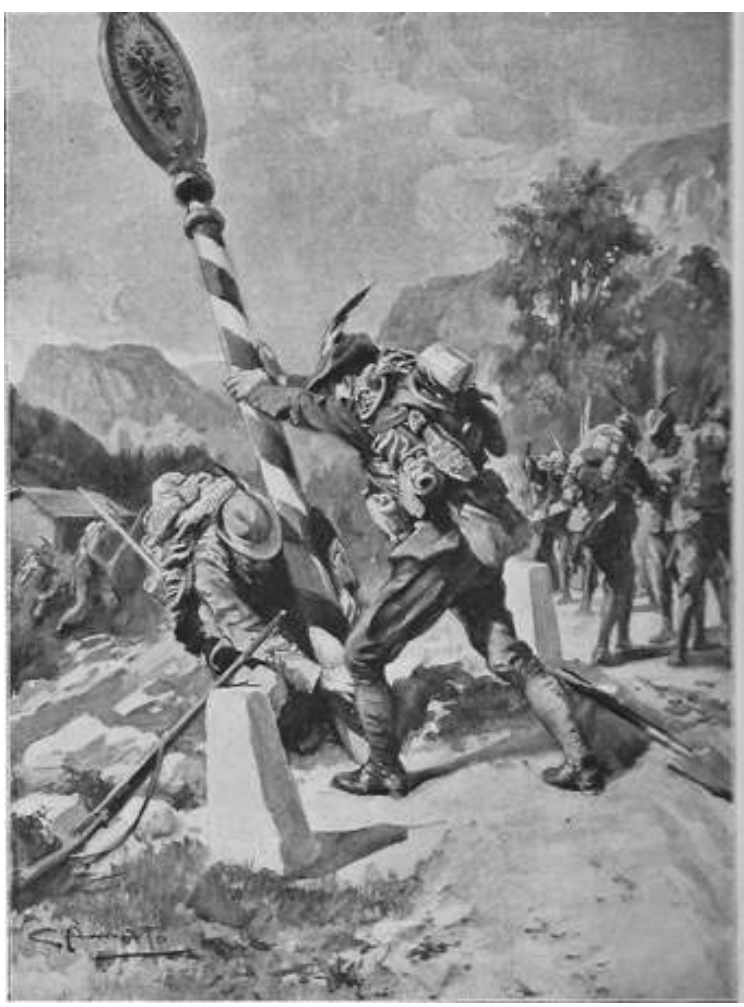

Dessin communiqué par l'Illustrazione italiana $»^{31}$

La Domenica del Corriere, supplément illustré du grand quotidien Il Corriere della Sera, montra le chasseur alpin prenant une aiguille escarpée, menant des opérations de commando, réalisant des exploits sportifs. La photographie et le dessin figeaient dans l'inconscient collectif tous les codes de l'alpino courageux et audacieux ainsi que la guerre en montagne dans sa dureté minérale et sa beauté mortifère. Dans la littérature de guerre, le combat en montagne nourrit les fantasmes d'un lectorat citadin en quête de sensations fortes et d'actes spectaculaires. Il devint plus important que celui mettant en scène les aviateurs "pourtant considérés comme les nouveaux chevaliers et les protagonistes, dans toute l'Europe, de la nouvelle mythologie guerrière $~^{32}$. Des œuvres comme Sott'la Naja. Vita e guerra d'alpini (En service. Vie et guerre de chasseurs alpins) de Mario Mariani, publié en $1916^{33}$, Con me e con gli alpini ${ }^{34}$ (Avec moi et avec les alpini) de Piero Jahier, publié en 1918-1919, Le scarpe al sole (Les chaussures au soleil) de Paolo Monelli, publié en $1921^{35}$, exaltèrent le bon sens paysan, le courage stoïque, la patience, la loyauté, la discipline, le sens du sacrifice, l'altruisme désintéressé et la camaraderie solidaire, autant de valeurs associées aux clichés de la vie austère en montagne. De la même manière, cette littérature reprenait le thème romantique du mythe de la montagne berceau de la régénération ou de la redécouverte des qualités physiques et mentales de l'homme. La recrue transformée en guerrier montagnard échappait à la vie décadente et malsaine de la ville après un rite initiatique et se sacrifiait pour la patrie et ses camarades.

18 Le cinéma s'empara également du mythe de l'alpino. Le film : Maciste Alpino sorti en 1916, produit par la société turinoise Itala Film et réalisé par Luigi Maggi et Giovanni Pastrone connut un énorme succès au point de devenir l'une des œuvres les plus 
représentatives de l'ensemble de la production culturelle italienne de guerre. Maciste, un robuste chasseur alpin, joué par Bartolomeo Pagano, incarna le héros capable, seul, de tenir en respect des Autrichiens sur fond d'actions spectaculaires d'exploits sportifs en haute montagne. Or le film devint un «formidable instrument de divertissement à vocation propagandiste, projeté également aux soldats lors de leur départ pour le front $\rrbracket^{36}$. Les films réalistes qui s'imposèrent à partir de 1916 exploitèrent également le paysage extraordinaire fourni par les crêtes, les pics et les aiguilles alpines. Luca Comerio réalisa ainsi La guerra d'Italia a 3000 metri sull'Adamello qui eut un gros succès en salle.

19 La force du mythe alpino finit par s'imposer à l'étranger pour symboliser le combat mené sur le front italien, en oubliant le Carso et la Bainsizza. En France, par exemple, L'Illustration publia la photographie d'alpini à l'assaut d'une aiguille. Il fallait en effet illustrer la prise du Monte Nero. Or la légende du journal français confortait la création du mythe des alpini de ce côté des Alpes: "L'attaque du Monte Nero [...] fut d'une hardiesse sans précédent. [...] Les hommes de quelques détachements ôtèrent même leurs souliers, et c'est les pieds nus qu'ils terminèrent, dans l'obscurité, une excursion presque incroyable, qui les mena à quelques mètres des retranchements autrichiens. Ceux-ci furent enlevés à la grenade et à la baïonnette ${ }^{37}$.

20 Après la guerre, le fascisme trouva dans le chasseur alpin, l'homme-guerrier dans toute sa dimension sacrificielle et héroïque. L'alpino, indiscipliné mais doté d'une sens aiguë du devoir, solidaire dans le combat mais en quête d'exploit individuel pour accomplir sa mission, courageux et dominant la nature hostile, gardien d'une Italie régénérée par la guerre, donnait une image conforme au discours idéologique du fascisme. Symbole d'une armée italienne conquérante, il occultait l'image du soldat fantassin résigné, passif et caporettiste ainsi que les hécatombes du Carso et de la Bainsizza et le désastre de Caporetto dont la honte rejaillissait sur l'Italie monarchique libérale. Ce n'est pas un hasard si le roman Piccolo alpino, du fasciste Salvator Gotta publié en 1926, servit d'ouvrage de référence pour les mouvements de jeunesses fascistes et fut porté à l'écran en 1940 par Oreste Biancoli ${ }^{38}$. L'histoire mettait en scène un jeune enfant qui après avoir suivi un alpino durant la guerre, avait été fait prisonnier par les Autrichiens, avant de réussir à s'échapper pour rejoindre les alpini. Naturellement, dans l'entre-deux-guerres, le fascisme sacralisa les lieux de ces combats transfigurés.

21 Si chaque village alpin a son monument aux morts lourdement chargé des noms des chasseurs alpins morts au cours de la Grande Guerre, les sanctuaires-ossuaires jalonnent aussi les champs de bataille du front montagneux. La guerre «blanche » eut ainsi ses lieux de mémoire sacralisés : l'Adamello, Asiago, le Pasubio, déclaré en 1922 Zone sacrée de la Patrie comme le monte Grappa, l'Ortigara et le col di Lana, et encore le Cadore avec le sanctuaire de Pocol, installé près de Cortina d'Ampezzo, construit en 1935. Ces lieux font encore aujourd'hui l'objet de circuits touristiques alpins aménagés dans des sortes d'écomusées de la Grande Guerre où les caractères de la guerre blanche furent conservés ou reconstitués. Un Sentier de la Paix, long de $520 \mathrm{~km}$, fut ainsi aménagé dans les années 1980 et permit la réhabilitation des forts de montagne austrohongrois et italiens. Ainsi la route des tunnels du mont Pasubio, réalisée en 1917 à 2000 $m$ d'altitude pour ravitailler les troupes italiennes en empruntant un versant à l'abri des Autrichiens offre au touriste l'occasion de longer sur six km les pentes abruptes et accidentées de la montagne. Au pied du Pasubio, un refuge à $1930 \mathrm{~m}$. d'altitude, nommé 
Achille Papa en hommage au commandant de la Brigade Liguria qui combattit sur le Pasubio et sur le plateau d'Asiago se trouve à l'endroit même des baraquements de la Grande Guerre et reste la propriété du Club alpin italien ${ }^{39}$. Ailleurs, dans les Cinque Torri dominées par le Tofana, un écomusée a été reconstitué d'après les photos de l'époque, avec des reproductions à l'échelle 1: 1 d'armes et de soldats, des tranchées, des tunnels, des baraquements et des nids d'artillerie et de mitrailleuses ${ }^{40}$.

\section{CONCLUSION}

La mémoire de la guerre blanche apparaît ainsi fortement concurrentielle de celle du Carso et de la Bainsizza même si cette dernière l'emporte dans le cœur des Italiens (chaque année, à l'occasion du 4-novembre, le président de la République ou le chef du gouvernement se rend ainsi au sanctuaire de Redipuglia, près de Gorizia!). Mais hors d'Italie, en France notamment, c'est bien la guerre blanche qui finit par occulter toutes les autres formes de guerre italienne. Il est vrai que la $47^{\mathrm{e}}$ division d'infanterie française, formée de chasseurs alpins aguerris dans les Vosges en 1914-1915, sut se distinguer en prenant le Monte Tomba le 30 décembre 1917 dans le secteur du Monte Grappa, et une plaque y a été posée par l'amicale du $22^{\mathrm{e}}$ Bataillon de Chasseur Alpin en 1980. Mais le documentaire historique «Apocalypse. La première guerre mondiale » réalisé par Daniel Costelle et Isabelle Clarke, diffusé sur France 2 du 18 mars au $1^{\mathrm{er}}$ avril 2014 en cinq épisodes dans le cadre du centenaire de la grande Guerre constitue un exemple saisissant ${ }^{41}$. À partir d'archives audiovisuelles colorisées, les réalisateurs évoquèrent ainsi, avec talent, les grands épisodes de ce conflit mondial. Mais les quelques minutes consacrées à l'Italie illustrèrent la guerre non par des images du Carso et de la Bainsizza mais par les exploits sportifs des Alpini et les paysages des Dolomites. Au fond, comme le déclara au journal Le Monde, Fabrice Puchault, responsable des documentaires sur France 2: «Isabelle Clarke et Daniel Costelle ont construit un récit, et leur point de vue n'est pas celui de deux historiens mais de deux cinéastes $»^{42}$. Plus que jamais, aujourd'hui comme hier, la guerre «blanche»des Italiens durant la Grande Guerre renvoya aux enjeux complexes d'une guerre fantasmée et fascinante dont l'adaptation, l'exploitation et la représentation furent souvent bien éloignée d'une réalité plus sombre et moins glorieuse.

\section{NOTES}

1. Voir en particulier Scala Edoardo, 1955, Storia delle fanterie italiane. Vol. 8 : Gli Alpini, Rome, SME-US, XXXI-742 p. ; Viazzi Luciano, 1978, Gli Alpini 1872-1945, Rome, Ciarrapico, 301 p. ; Paoletti Ciro, 2015, « L'adaptation de l'armée italienne aux conditions du combat en montagne entre 1915 et 1918 », RHA, « Guerre en montagne », n²78, pp. 17-24.

2. L'Illustration, $\mathrm{n}^{\circ} 3769,29$ mai 1915, p. 559

3. Rabot Charles, 29 mai 1915, «Vue panoramique du nouveau front de guerre, de la frontière suisse à l'Istrie », L'Illustration, n 3769, pp. 552-554. 
4. Minola Mauro et Beppe Ronco, 1998, Fortificazioni nell'arco alpino. L'evoluzione delle opere defensive tra XVIII e XX secolo, Quaderni di cultura alpina, 61-62, Scarmagno (Turin), Priuli \& Verlucca ed., 1998, p. 19 n. 16 et 18, 55 et 68. Mauro Minola, Beppe Ronco, Fortificazioni di montagna. Dal Gran S. Bernardo al Tonale e la cintura difensiva Svizzera, Varese, Macchione ed., 1998, pp. 5-8, 15 et 24. Maurizio Ruffo, L'Italia nella Triplice Alleanza, i piani operativi dello Stato Maggiore verso l'AustriaUngheria dal 1885 al 1915, Rome, SME-US, p. 84, 87 et 92-93, 97-100.

5. Regolamento d'istruzione e di servizio interno per la Fanteria, Rome, Voghera, 1892.

6. Stefani Filippo, 1984, La storia della dottrina e degli ordinamenti dell'esercito italiano, vol. 1 : «Dall'esercito piemontese all'esercito di Vittorio Veneto", Rome, SME-US, pp. 416-427 et 488-493.

7. Cadorna Luigi, 1929, « Memoria riassuntiva circa un'azione offensiva verso la Monarchia a. u. durante l'attuale conflagrazione europea. Possibili obiettivi. Presumibili operazioni da svolgersi », ministère de la Guerre, SME-US, L'esercito italiano nella Grande Guerra 1915-1918, vol. 2bis : Le operazioni del 1915. Documenti, Rome, Istituto poligrafico dello Stato, allegato ${ }^{\circ} 1, \mathrm{pp} .1-8$.

8. Cadorna Luigi, 1980, "Criteri generali per la sistemazione difensiva in montagna ", 29 avril 1917, circulaire $n^{\circ}$ 2324, ministère de la Guerre, SME-US, L'esercito italiano..., vol. 6, t. 2 : « Le istruzioni tattiche del capo di Stato maggiore dell'Esercito, 1917-1918 », Rome, Istituto poligrafico dello Stato, allegato $\mathrm{n}^{\circ} 34$, pp. 153-158.

9. Ministère de la Guerre, SME-US, L'esercito italiano..., vol. 2-1 : Le operazioni del 1915 (Narrazione), Rome, Istituto poligrafico dello Stato, 1929, pp. 118-122.

10. Cadorna Luigi, 1932, Norme complementari all'Istruzione sui lavori del camp di battaglia, Parte II : «Lavori del campo di battaglia: Ripari in montagna », circulaire $n^{\circ}$ 250, Rome, 10 février 1915, ministère de la Guerre, SME-US, L'esercito italiano..., vol. 6, t. 1 : « Le istruzioni tattiche del capo di Stato maggiore dell'Esercito degli anni 1914-1915-1916», Rome, Istituto poligrafico dello Stato, allegato $\mathrm{n}^{\circ} 2$, pp. 39-40.

11. Luigi Cadorna, Fascicolo contenente «Criteri d'impiego d'artiglieria », zone de guerre, avril 1916, ministère de la Guerre, SME-US, L'esercito italiano..., vol. 6, t. 1, op. cit., allegato $\mathrm{n}^{\circ} 22$, Capo VII : Postazione delle batterie, § e et f, p. 252.

12. Ibid., p. 75.

13. Id., p. 124.

14. Ortali Alfredo, 2008, Dalla Romagna alla Marmolada per un ideale repubblicano. Diario di guerra, présenté et commenté par Andrea de Bernardin, Udine, Gaspari ed., pp. 25-60.

15. Lettre de Gelasio Gaetano, à son père, retrouvé par Luciano Viazzi, Col di Lana. Monte di fuoco, Milan, Mursia, 1985, pp. 140-141.

16. Cité dans Fabi Lucio, 1995, Uomini, armi e campi di battaglia della Grande Guerra. Fronte italiano, 1915-1918, Milan, Mursia, p. 133.

17. Ibid., p. 119.

18. Id., p. 120.

19. Minsitère de la Guerre, SME-US, 1936, L'esercito italiano..., vol. 3 : Le operazioni del 1916, t. 2 : «Offensiva austriaca e controffensiva italiana nel Trentino. Contemporanee operazioni sul resto della fronte (maggio-luglio 1916), (Narrazione) », Rome, Istituto poligrafico dello Stato, pp. 37-205 et 209-252.

20. Ministère de la Guerre, SME-US, 1954, L'esercito italiano..., vol. 4 : Le operazioni del 1917, t. 2 : «Gli avvenimenti dal giugno al settembre (Narrazione) », Rome, Istituto poligrafico dello Stato, pp. 57-150.

21. Peffafato Enzo et Laurent, Verney Jean-Pierre, 2006, L'Italie en guerre. 1915-1918, Soteca, 14-18 éditions, pp. 61-62. Isnenghi Mario, Rochat Giorgio, 2000, La Grande Guerra, 1914-1918, Milan, RCS S.p.A., pp. 182-183.

22. Mario Isnenghi, Giorgio Rochat, La Grande Guerra..., op. cit., pp. 201-202. 
23. Ministère de la Guerre, SME-US, 1931, L'esercito italiano..., vol. 3 : Le operazioni del 1916, t. 1 : «Gli avvennimenti invernali (Narrazione) », Rome, Istituto poligrafico dello Stato, pp. 330-337.

24. Enzo et Laurent Peffafato, Jean-Pierre Verney, L'Italie en guerre, op. cit., pp. 73-74.

25. Commissariato generale Onoranze Caduti in Guerra, Sacrari militari della prima guerra mondiale, 1915-1918. Asiago. Pasubio, ministère de la Défense, Rome, 1984, pp. 37-40.

26. Cité par Lucio Fabi, Uomini, armi..., op. cit., pp. 125-128.

27. Stato Maggiore dell'Esercito, La Grande Guerra..., op. cit., pp. 7-12.

28. Ibidem, p. 20.

29. Rochat Giorgio, Isnenghi Mario, La Grande Guerra..., op. cit., p. 160.

30. Mondini Marco, Alpini. Parole e immagini di un mito guerriero, 2008, Rome-Bari, Laterza, pp. 63-77.

31. L'Illustration, $\mathrm{n}^{\circ} 3771,12$ juin 1915, p. 608.

32. Mondini Marco, 2014, La guerra italiana. Partire, raccontare, tornare, 1914-1918, Bologne, il Mulino, p. 194.

33. Mariani Mario, 1918, Sott'la Naja. Vita e guerra d'alpini, Milan, Sonzogno, 221 p.

34. Jahier Piero, 1920, Con me e con gli alpini, Rome, La Voce, soc. an. ed., 196 p.

35. Monelli Paolo, 1921, Le scarpe al sole : cronaca di gaie e di tristi avventure d'alpini, di muli e di vino, Bologne, L. Cappelli, 227 p.

36. Faccioli Alessandro, 2014, «Pellicole sul fronte : il cinema e la guerra », Dizionario storico della Prima guerra mondiale, sous la direction de Nicola Labanca, Rome-Bari, Laterza, p. 383. Id., « Rulli di guerra nel cinema muto ", Gli italiani in guerra. Conflitti, identità, memorie, dal Risorgimento ai nostri giorni, sous la direction de Mario Isnenghi, vol. III : « La Grande Guerra : dall'intervento alla «vittoria inutilata », sous la direction de Mario Isnenghi et Daniele Ceschin, Turin, UTET, 2008, t. 2, pp. 871-873.

37. « Les “Alpini“ à plume d'aigle », L'Illustration, n 3777, 24 juillet 1915, p. 97.

38. Gotta Salvator, 1926, Piccolo alpino, Milan Mondadori, 255 p.

39. Ibid., pp. 75-80

40. Id., pp. 75-80, 81-87, 94-105 et 115-120.

41. Costelle Daniel, Clarke Isabelle (réalisateurs), 2014, Apocalypse. La Première Guerre mondiale, France Télévision Distribution, coffret de trois DVD, $295 \mathrm{mn}$.

42. Psenny Daniel, «"Apocalypse“ ravive la Grande Guerre en couleur », Le Monde.fr, 14 mars 2014.

\section{RÉSUMÉS}

Au cours de la Grande Guerre, sur le front italien, une guerre montagneuse «blanche » se déroula dans les Alpes, entre 2000 et 3000 mètres d'altitude. Pour s'adapter aux contraintes hostiles du milieu, le Grand Quartier Général italien adopta une tactique à la fois défensive et offensive, générant ainsi une guerre polymorphe faite de coups de main, de tranchées, de mines parfois même dans les glaciers. Mais les combats, particulièrement éprouvants et meurtriers, nourrirent une représentation fantasmée iconographique, permirent une transfiguration héroïsante des combattants (dont les chasseurs alpins) et finirent par sacraliser les champs de bataille en lieux de mémoire à l'historicisation différenciée. 
During the First World War, to the italian front, a « white » mountainous war developped into the Alps, beetween 2000 and 3000 meters. To adapt to the hostile constraints of the environment, the italian General Head Quarted adopted a defensive and offensive tactic. Doing this, generated a polymorphous war with raids, trenchs, saps, even sometimes into glaciers. But the actions of war, particulary suffering and bloodies, improved an iconographical fantasized representation, allowed a soldiers 's transfiguration into heros (among which were the alpine chasseurs) and, finally, regarded as sacred the battlefields transformed in places of memory with a differentiated historicisation.

INDEX

Keywords : Alps, First World War, alpine chasseurs, memory of war, places of memory, heromaking

Mots-clés : Alpes, Grande Guerre, chasseurs alpins, mémoire de guerre, lieux de mémoire, fabrique des héros

\section{AUTEUR}

\section{HUBERT HEYRIÈS}

Professeur en Histoire contemporaine à l'Université de Montpellier 3 